Ophthalmologe 2016 $\cdot 113: 570-580$

DOI 10.1007/s00347-016-0224-x

Online publiziert: 11. Februar 2016

(c) Die Autor(en) 2016. Dieser Artikel ist auf

Springerlink.com mit Open Access verfügbar

CrossMark

B. Heimes ${ }^{1} \cdot$ T. Schick ${ }^{2}$ C.K. Brinkmann ${ }^{3} \cdot$ A. Wiedon ${ }^{4}$ B. Haegele ${ }^{4}$ B. Kirchhof ${ }^{2}$. F.G. Holz ${ }^{3}$. D. Pauleikhoff' - F. Ziemssen ${ }^{5} \cdot$ S. Liakopoulos ${ }^{2} \cdot$ G. Spital ${ }^{1}$. S. Schmitz-Valckenberg ${ }^{3}$

${ }^{1}$ M3 Reading Center, Augenabteilung am St. Franziskus-Hospital Münster, Münster, Deutschland

${ }^{2}$ Cologne Image Reading Center, Zentrum für Augenheilkunde, Uniklinik Köln, Köln, Deutschland

${ }^{3}$ GRADE Reading Center, Universitäts-Augenklinik Bonn, Bonn, Deutschland

${ }^{4}$ Novartis Pharma GmbH Nürnberg, Nürnberg, Deutschland

${ }^{5}$ Universitäts-Augenklinik Tübingen, Tübingen, Deutschland

\title{
Design des ORCA-Moduls der OCEAN-Studie
}

\section{Evaluierung der SD-OCT-Befunderhebung in der täglichen Praxisroutine}

Retinologische Gesellschaft (RG) und die Deutsche Ophthalmologische Gesellschaft (DOG) empfehlen in den aktuellen Stellungnahmen [6-8] bei Vorliegen einer aktiven choroidalen Neovaskularisation (CNV) oder eines Makulaödems mit fovealer Beteiligung durch RVV oder Diabetes mellitus als First-line-Therapie die intravitreale Injektion von VEGFHemmern. Zur Diagnostik und Verlaufskontrolle sollte neben der Funduskopie und der Fluoreszeinangiographie primär die hochauflösende SD-OCT verwendet werden.

Die Verbesserung der Sehkraft der Patienten im klinischen Alltag bleibt jedoch häufig hinter den Ergebnissen prospektiver, kontrollierter, randomisierter Studien zurück [11, 18, 22]. Eine mögliche Erklärung hierfür ist, dass es im klinischen Alltag zu einer Unterbehandlung der Patienten kommt. In einer deutschlandweiten Untersuchung konnten Finger et al. [11] zeigen, dass im ersten Jahr der Behandlung einer nvAMD im Mittel nur 4,3 Injektionen gegeben wurden und nur ein Drittel der Patienten 4 oder mehr Injektionen erhielten [21]. In einer randomisierten Vergleichsstudie zwischen Bevacizumab und Ranibizumab zur Behandlung der nvAMD (Comparison of Age-Related Macular Degeneration Treatments Trials, CATT) wurden in einem bedarfsorientierten Wieder- behandlungsschema gute funktionelle Ergebnisse mit im Durchschnitt ca. 7 Injektionen erreicht [5]. Die Ursachen für die geringere Anzahl an Injektionen in Deutschland sind sicherlich vielfältig und $u$. a. auch in gesundheitspolitischen Rahmenbedingungen zu vermuten.

Die nichtinterventionelle AURAStudie hat den Behandlungsalltag von nvAMD-Patienten europaweit untersucht und gezeigt, dass die reale Versorgungssituation in Großbritannien oder den Niederlanden mit den Ergebnissen der CATT-Studie vergleichbar ist [14]. Trotz des europaweit einheitlichen Labels waren dagegen Deutschland und Italien durch ungünstigere Visusresultate unter Therapie gekennzeichnet. Hinzu kommt, dass Komorbiditäten der häufig älteren Patienten in der Praxis zu Mobilitätsproblemen führen und somit häufige und regelmäßige Augenarztbesuche erschweren können. Ein weiterer möglicher Faktor könnte ein Übersehen von Aktivitätskriterien durch fehlende oder fehlerhaft interpretierte Bildgebung darstellen.

Die Stellungnahmen der Fachgesellschaften empfehlen im Rahmen der Anti-VEGF-Therapie die Einbeziehung morphologischer Kriterien, wie sie mittels SD-OCT erhoben werden können. Die Interpretation der zum Teil komplexen Veränderungen auf SD-OCT-Scans sowie die Entscheidungsfindung zur 
(Wieder-)Behandlung von Patienten mit chorioretinalen vaskulären Pathologien stellt eine Herausforderung dar. In der CATT-Studie wurde gezeigt, dass die Auswertung der Befunde der zentralen Reading-Center bezüglich der Aktivitätskriterien in nur ca. $69 \%$ mit der Therapieentscheidung der Studienzentren übereinstimmte. In $30 \%$ bestand eine Diskrepanz, die in $95 \%$ zu einer Unterbehandlung der Studienpatienten führte [16]. Diese mögliche Unterbehandlung aufgrund inkorrekt interpretierter Bildbefunde ist eine weitere mögliche Ursache dafür, dass die Ergebnisse in der Praxis hinter denen der klinischen Studien zurückbleiben.

Eine genaue Analyse der Qualität der SD-OCT-Auswertung im Hinblick auf die verschiedenen Aktivitäts- und Wiederbehandlungskriterien in Deutschland erscheint damit wünschenswert, um Schwierigkeiten $\mathrm{zu}$ identifizieren und ggf. durch gezielte Formulierung von Standards die Qualität der Patientenversorgung zu optimieren.

Die nichtinterventionelle OCEAN (Observation of treatment patterns with Lucentis and real life ophthalmic monitoring, including optional OCT in approved indications)-Studie untersucht die Behandlung von nvAMD, Makulaödemen aufgrund diabetischer Retinopathie und venöser Verschlüsse sowie CNV bei pathologischer Myopie (PM) unter Behandlung mit Ranibizumab im Versorgungsalltag (ClinicalTrials. gov: NCT02194803). Dabei sollen Behandlungs- und Beobachtungsintervalle dokumentiert und der Nutzen der Behandlung in Relation zum Visus, zur Lebensqualität der Patienten sowie zum morphologischen Netzhautbefund analysiert werden.

Das zusätzliche Modul ORCA der OCEAN-Studie evaluiert die Befundauswertung von SD-OCT-Scans durch niedergelassene Ophthalmologen und Klinikärzte im Vergleich zur standardisierten Auswertung durch 3 spezialisierte Reading-Center [Cologne Image Reading Center (CIRCL), Zentrum für Augenheilkunde, Uniklinik Köln; GRADE Reading Center, UniversitätsAugenklinik Bonn; $\mathrm{M}^{3}$ Reading Center, Augenabteilung am St. FranziskusHospital Münster]. Mit der ORCA-
Studie werden der Status quo der SDOCT-Auswertung sowie (Wieder-)Behandlungsentscheidungen in der täglichen Praxisroutine evaluiert. Außerdem sollen der Einfluss einzelner Befunde auf die Therapieentscheidung sowie die Bedeutung von Referenzwerten spezifiziert werden.

\section{Patienten und Methoden}

In der OCEAN-Studie werden über einen Zeitraum von 24 Monaten Patienten mit nvAMD, Makulaödem bei DMÖ, RVV und CNV bei PM unter Behandlung mit Ranibizumab in der täglichen Routine beobachtet (ClinicalTrials. gov: NCT02194803). Dabei wurden die Teilnahme von 600 Augenabteilungen und Praxen und der Einschluss von 6000 Patienten angestrebt. Voraussetzung für den Einschluss als Studienzentrum in das ORCA-Modul ist die Möglichkeit der Durchführung von SD-OCTUntersuchungen. Nach Registrierung und OCT-Zertifizierung konnten Patienten, die unter einer der folgenden Erkrankungen leiden, in das ORCA-Modul eingeschlossen werden:

- aktive neovaskuläre altersabhängige Makuladegeneration (nvAMD),

- Visusbeeinträchtigung infolge eines diabetischen Makulaödems (DMÖ),

- Visusbeeinträchtigung aufgrund eines Makulaödems infolge eines retinalen venösen Verschlusses (RVV).

Geeignete Patienten durften in den letzten 3 Monaten keine intravitreale Injektion mit Anti-VEGF-Medikamenten erhalten haben. Eine Vorbehandlung mit intravitrealen Steroiden ist ebenfalls nicht zulässig.

Um eine qualitativ hochwertige und standardisierte Auswertung der bildgebenden Befunde gewährleisten zu können, haben die 3 beteiligten ReadingCenter (CIRCL, GRADE, $\mathrm{M}^{3}$ ) anhand internationaler Standards in einem Konsens qualitative Anforderungen für die Fundusfotografie, Angiographie sowie verschiedene SD-OCT-Instrumente erarbeitet. Empfehlungen zu Aufnahmeart und Einstellungen sowie Protokolle für SD-OCT, Farbfotografie und Angiographie sind im ORCA-Handbuch zu- sammengefasst. Jede interessierte Praxis/ Augenabteilung wurde vor Aufnahme in die Studie anhand von Beispielaufnahmen hinsichtlich der Qualität ihrer SD-OCT-Aufnahmen geprüft. Waren die definierten Qualitätsstandards gewährleistet, so wurde das Zentrum für die Studienteilnahme freigegeben (Zertifizierung). Für die Farbfotografie und die Angiographie wurden Empfehlungen durch die Reading-Center formuliert; allerdings erfolgte keine benutzerspezifische Zertifizierung für diese beiden Modalitäten an den teilnehmenden Zentren.

Ein speziell entwickelter Fragebogen enthält Fragen zu allen für eine Entscheidung zur (Wieder-)Behandlung relevanten Parameter. Diese werden unabhängig sowohl vom Studienzentrum als auch von den Reading-Centern beantwortet. Die Reading-Center sind hierbei gegenüber den Antworten der Studienzentren verblindet. Die Aufnahmen der Patienten werden unabhängig durch jeweils 2 speziell trainierte und zertifizierte ärztliche Reader aus unterschiedlichen Reading-Centern befundet. Im Anschluss wird der Fall zusätzlich von einem Senior-Reader geprüft (• Abb. 1).

Abschließend werden die individuellen SD-OCT-Befundungen der Studienzentren den standardisierten Befundungen der Reading-Center gegenübergestellt und hinsichtlich Übereinstimmungen und Abweichungen einzelner Parameter analysiert.

\section{Behandlungs- und Abbruchkriterien}

Für alle zugelassenen Indikationen von Ranibizumab haben die RG, die DOG und der BVA in einem Konsens eine Empfehlung zur Diagnostik und Therapie formuliert. $\mathrm{Zu}$ allen genannten Erkrankungen werden bedarfsorientierte Behandlungsschemata empfohlen, um eine Überbehandlung zu vermeiden. Die Wiederbehandlung orientiert sich hierbei hauptsächlich an Aktivitätszeichen auf bildgebenden Befunden. Einer sorgfältigen Diagnostik kommt somit eine besondere Bedeutung zu.

Zur initialen Diagnostik werden für alle Indikationen folgende Unter- 
Ophthalmologe 2016·113:570-580 DOI 10.1007/s00347-016-0224-x

(c) Die Autor(en) 2016. Dieser Artikel ist auf Springerlink.com mit Open Access verfügbar

B. Heimes - T. Schick · C.K. Brinkmann · A. Wiedon · B. Haegele · B. Kirchhof · F.G. Holz · D. Pauleikhoff · F. Ziemssen · S. Liakopoulos · G. Spital · S. Schmitz-Valckenberg

\section{Design des ORCA-Moduls der OCEAN-Studie. Evaluierung der SD- OCT-Befunderhebung in der täglichen Praxisroutine}

Zusammenfassung

Hintergrund. Die Prävalenz von Er-

blindungen im Sinne des Gesetzes konnte durch die Einführung der intravitrealen

Anti-VEGF („vascular endothelial growth factor")-Therapie reduziert werden. Da die Behandlung bedarfsgesteuert $u$. a. anhand morphologischer Kriterien erfolgt, kommt der bildgebenden Diagnostik ein besonderer Stellenwert zu. Die nichtinterventionelle OCEAN-Studie untersucht die Behandlung mit Ranibizumab im klinischen Alltag. In einer Subgruppe analysiert das ORCAModul die Befundung von Spectral-Domain optischer Kohärenztomographie (SD-OCT)Scans durch die behandelnden Ärzte. Methoden. Über 24 Monate werden die Daten von Patienten mit exsudativer altersabhängiger Makuladegeneration (AMD), einem Makulaödem durch retinalen Venenverschluss oder Diabetes mellitus erfasst, die intravitreale Injektionen mit Ranibizumab erhalten. Informationen über Untersuchungen, Visus, Behandlungen sowie bildgebende Verfahren werden anhand eines Fragebogens dokumentiert. Durchgeführte SD-OCT-Scans, Fluoreszeinangiographien und Fundusfotografien werden unabhängig voneinander durch den Augenarzt des Studienzentrums sowie durch 3 Reading-Center (CIRCL Köln, GRADE Bonn, M3 Münster) befundet. Die automatische Messung der Netzhautdicke durch die Herstellersoftware wird überprüft und ggf. manuell korrigiert. Es erfolgt eine qualitative Auswertung im Hinblick auf morphologische Kriterien für eine (Wieder-) Behandlung.
Schlussfolgerungen. Eine sorgfältige Beurteilung der SD-OCT-Aufnahmen im Verlauf unter einer Anti-VEGF-Therapie bildet die Grundlage für eine bestmögliche bedarfsorientierte Behandlung. Die Überprüfung der Befunderhebungsqualität in der täglichen Praxis kann hierbei mögliche Schwachstellen aufzeigen, gezieltes Training ermöglichen und somit die Behandlung der Patienten optimieren.

\section{Schlüsselwörter}

Optische Kohärenztomographie . Altersabhängige Makuladegeneration . Diabetisches Makulaödem · Retinaler Venenverschluss · Anti-VEGF

\section{Design of the ORCA module in the OCEAN study. Evaluation of SD-OCT results in daily routine practice}

\section{Abstract}

Background. The prevalence of blindness as defined by law could be reduced by the introduction of anti-vascular endothelial growth factor (VEGF) therapy. Because the treatment is governed by patient needs, mostly using morphological criteria, imaging diagnostics are of particular importance. The non-interventional OCEAN study investigates the treatment with ranibizumab in the clinical routine practice. In a subgroup of patients the interpretation of spectral domain optical coherence tomography (SD-OCT) scans by the treating physicians will be analyzed (ORCA module).

Methods. Over a period of 24 months data from patients with exudative age-related macular degeneration (AMD), macular edema due to retinal vein occlusion or diabetes mellitus, who are receiving intravitreal injections of ranibizumab, will be assessed. Information on examinations, visual acuity, treatment and recordings from imaging techniques will be documented using a questionnaire. The SD-OCT scans, fluorescence angiography and fundus photography will be independently analyzed by the ophthalmologist of the study center and by three reading centers (CIRCL Cologne, GRADE Bonn and M3 Münster). Automated measurements of retinal thickness by the manufacturers' software will be checked and if necessary manually corrected. A qualitative interpretation in terms of morphological criteria for (further) treatment will be performed.

Conclusion. A thorough assessment of SDOCT images during anti-VEGF therapy provides the basis for the best possible needsoriented treatment regimen. The control of the quality of data from daily routine practice may indicate possible weaknesses allowing explicit training and therefore optimization of patient treatment.

\section{Keywords}

Optical coherence tomography . Age-related macular degeneration . Diabetic macular edema $\cdot$ Retinal vein occlusion · Anti-VEGF suchungen empfohlen: bestkorrigierter Visus, binokulare Ophthalmoskopie in Mydriasis, Fluoreszeinangiographie sowie ein SD-OCT-Volumenscan. Für die diabetische Retinopathie wird außerdem auf den Wert einer Fundusfotografie hingewiesen, bei retinalen venösen Verschlüssen besonders auf die Augeninnendruckmessung [6-8].

Alle Stellungsnahmen betonen, dass die Messung der Sehschärfe allein nicht für die Entscheidung einer (Wieder-)Behandlung ausschlaggebend sein sollte [6-8].

\section{Altersabhängige Makuladegeneration}

Verschiedene läsionsaktivitätbasierte Behandlungsschemata sind möglich [6]. In einem Pro-re-nata (PRN)-Algorithmus erfolgt nach initialer Aufsättigung eine Wiederbehandlung im Falle des Vorliegens einer Läsionsaktivität. Bei einem Treat-and-Extend (TE)-Schema erfolgt bei jeder Vorstellung eine Behandlung. Die Aktivitätskriterien entscheiden über die Dauer bis zur nächsten Visite, die bei Aktivität der Läsion verkürzt und bei
Stabilität verlängert wird. Sowohl für die PRN- als auch TE-Behandlungsstrategie gelten neben einer neuen sub- oder intraretinalen Blutung folgende OCTrelevante Aktivitätskriterien:

- Anwesenheit subretinaler Flüssigkeit im SD-OCT,

- Persistenz oder Zunahme der diffusen Netzhautverdickung im SDOCT,

- Zunahme intraretinaler zystoider Flüssigkeitsräume im SD-OCT, 


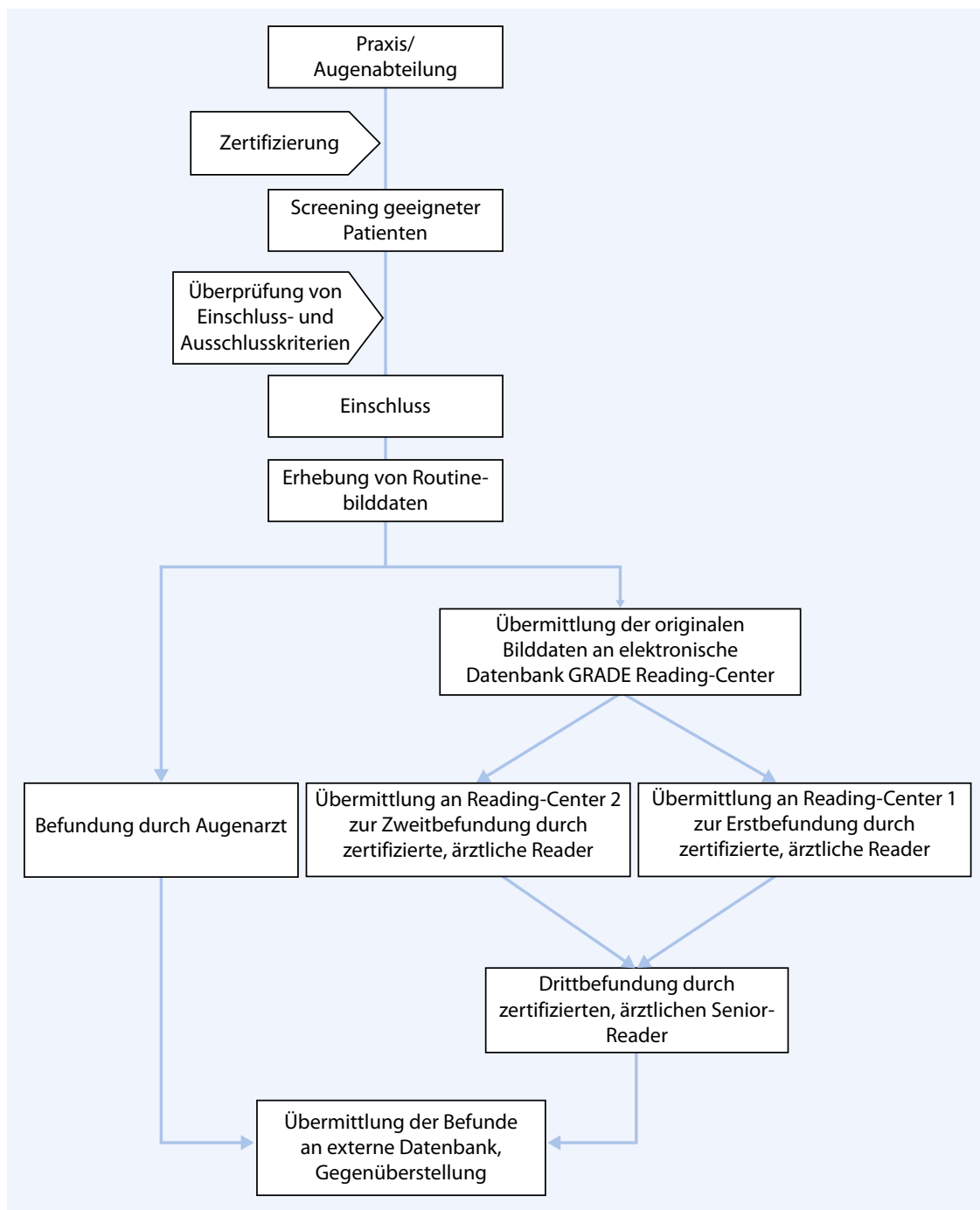

Abb. 1 A Flussdiagramm zum Ablauf des Readings der Bilddaten in der ORCA-Studie

- Zunahme einer serösen Pigmentepithelabhebung im SD-OCT [6].

Eine Sehschärfe $<0,05$, die durch eine relevante Atrophie der äußeren Netzhaut ( $\bullet$ Abb. 2a) oder eine fibrotische Umwandlung der CNV (• Abb. 2b) erklärt ist, kann ein Kriterium für einen Therapieabbruch darstellen [6].

Im Falle eines fehlenden Ansprechens auf die Therapie sollte geprüft werden, ob die Diagnose richtig gestellt wurde oder ob vorhandene zystoide Räume möglicherweise nicht durch eine aktive $\mathrm{CNV}$ verursacht werden. Dies kann z. B. bei chronischen zystoiden Räumen als Zeichen einer Gewebedestruktion [besonders im Bereich von RPE (retinales Pigmentepithel)-Atrophien und Fibrosen] vorkommen oder wenn eine

\section{Diabetische Makulopathie}

Für die diabetische Makulopathie ist eine initiale Fluoreszeinangiographie von großer Bedeutung, um Ischämien (im Bereich der Makula oder peripher) sowie Neovaskularisationen zu erkennen und eine Laserindikation stellen zu können. Die hohe Sensitivität der SD-OCTUntersuchung für die qualitative wie auch quantitative Erfassung intra- und subretinaler Flüssigkeit ist sowohl bei der Diagnosestellung als auch bei den Verlaufskontrollen hilfreich [8].

Für das klinisch signifikante DMÖ mit fovealer Beteiligung empfiehlt die aktuelle Stellungnahme eine bedarfsorientierte Behandlungsstrategie, die sich vornehmlich auf qualitative wie quantitative Befunde des SD-OCT stützt. Im Unterschied zur AMD sind quantitative Kriterien aufgeführt, da die automatische Segmentierung der OCT-Geräte bei rein retinalen Erkrankungen weniger Segmentierungsfehler aufweist und eine geringe Zunahme oder Abnahme der Dicke auf quantitativen Dickenkarten teils besser erkannt werden kann als bei der rein qualitativen Betrachtung der Scans. Vor Verwendung der quantitativen Werte ist es jedoch wichtig zu prüfen, ob die Segmentierungslinien sowie die Position des ETDRS-Grids korrekt sind.

Auch für das diabetische Makulaödem ist sowohl ein PRN- als auch ein TESchema etabliert $[8,17]$. Beide stützen sich auch hier auf SD-OCT-relevante Aktivitätskriterien.

Zeigt sich 4 Wochen nach der letzten Injektion ein Befund, der keinen weiteren positiven Behandlungsverlauf erwarten lässt, so sollte die Therapie abgebrochen werden (Abbruchkriterien). $\mathrm{Zu}$ diesen Abbruchkriterien zählen:

- ein Visus <0,05 (wenn keine Verbesserung zu erwarten ist, Ausnahme: z. B. akute Glaskörperblutung),

- eine irreversible Schädigung der Netzhaut durch zentrale Atrophie der äußeren Netzhaut oder ausgeprägte Ischämie [8].

Als Erfolgskriterien gelten hingegen eine Verbesserung der Sehschärfe auf 1,0 oder besser oder das Fehlen eines Ödems mit fovealer Beteiligung. In diesem Fall sollten keine weitere Injektionen erfolgen, jedoch 

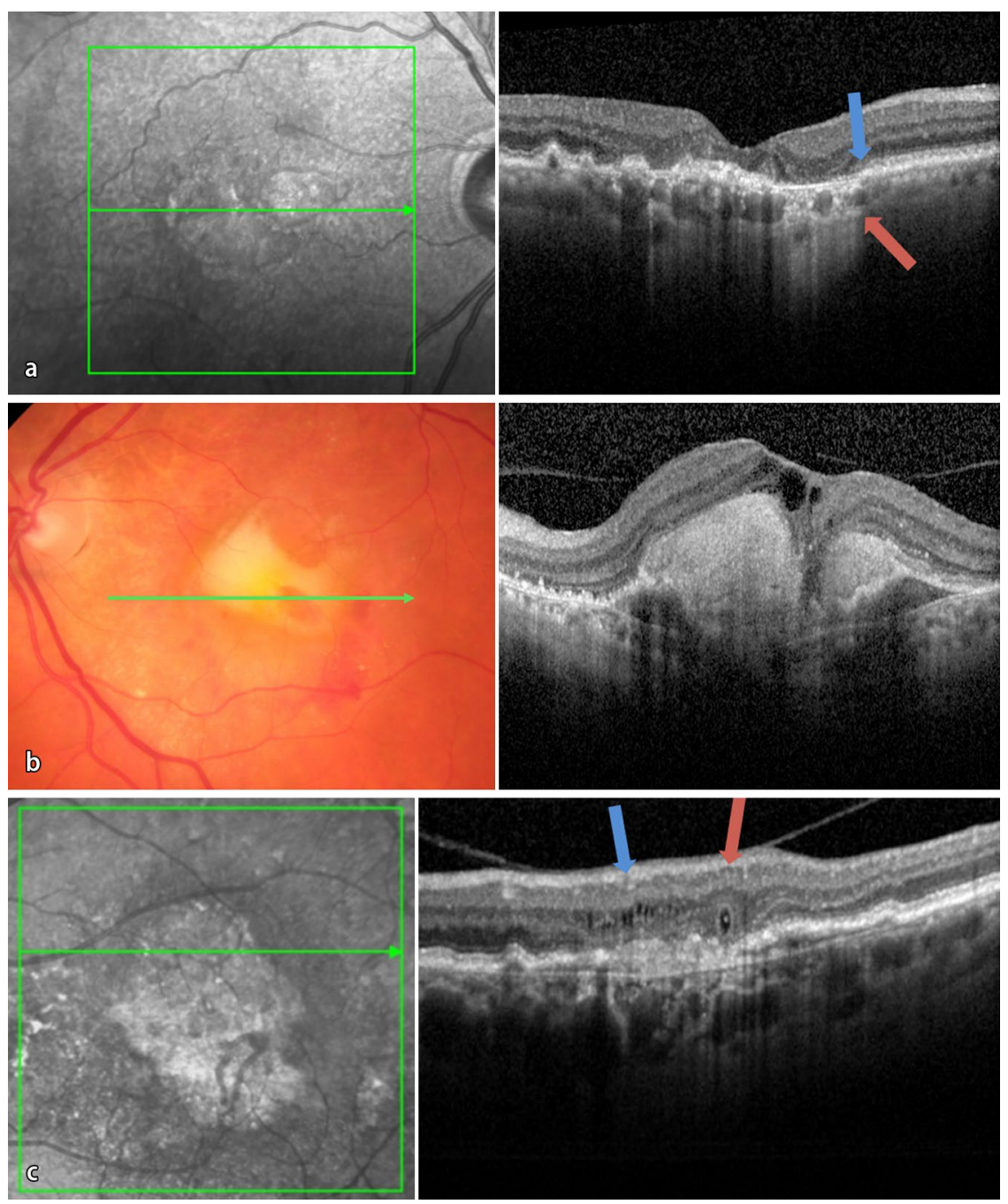

Abb. $2 \Delta$ a Beispiel-OCT für eine zentrale Atrophie mit diskontinuierlichen äußeren Banden [externe Grenzmembran, ellipsoide Zone und RPE (retinales Pigmentepithel)-Bande, blauer Pfeil] und verstärkter Transmission von Licht in die Aderhaut (roter Pfeil). b Beispiel einer deutlichen subretinalen fibrotischen Umwandlung mit wenig intraretinaler Flüssigkeit darüber im Vergleich zum Fundusfoto; aufgenommen mit Spectralis-OCT. c Ältere choroidale Neovaskularisationen mit Atrophie, subretinaler Fibrosierung " , $^{\prime}$ uter retinal tubulations" (ORT, roter Pfeil) und dezenten zystoiden Veränderungen (blauer Pfeil); aufgenommen mit Spectralis-OCT

über mindestens 6 Monate monatliche Kontrollen durchgeführt werden [8].

Liegt weder ein Abbruchkriterium noch ein Erfolgskriterium vor, so sollten die Wirksamkeitskriterien überprüft werden. $\mathrm{Zu}$ diesen zählen:

- eine Visusverbesserung um mindestens 1 Zeile oder 0,1 logMAR,

- ein relevanter Rückgang des

Makulaödems (um mindestens $10 \%$ des Ausgangswertes) [8].

In diesem Fall sollte die Therapie fortgesetzt werden. War die Therapie bisher nach diesen Kriterien nicht wirksam, so sollte eine Therapiepause mit 4-wöchent- als Therapie der ersten Wahl empfohlen. Diese orientiert sich vornehmlich an SDOCT-Kriterien. Alternativ kann auch eine intravitreale Steroidtherapie erwogen werden, wobei auf die Risiken einer Kataraktentwicklung und eines Glaukoms hingewiesen werden muss. Eine GridLaserkoagulation ist als Second-lineTherapie beschrieben [7].

In die Basisdiagnostik werden zur SD-OCT-Untersuchung zusätzlich eine Funduskopie und Angiographie empfohlen, um aktive Neovaskularisationen oder ischämische Areale zu identifizieren [7].

Auch für venöse Verschlüsse gibt es ein PRN-Schema und auch eine TE-Behandlungsstrategie $[7,18]$. Bei einer Sehschärfe unter 0,05 wird eine Therapie nur in Ausnahmefällen empfohlen, während für den Visus keine Obergrenze angegeben wird [7].

Die derzeit verbreitete Behandlungsmethode ist das PRN-Schema. Besteht bei der Kontrolluntersuchung mittels SDOCT ein verbessertes oder persistierendes Makulaödem mit angestiegenem Visus, so wird zu einer Behandlung geraten. Stellt sich hierin kein Ödem mehr dar oder zeigen sich Ödem und Visus trotz Therapie unverändert, so kann die Behandlung nachfolgend pausiert werden. Während einer Therapiepause sollten Kontrollen mittels SD-OCTUntersuchung für mindestens 6 Monate in 4-wöchentlichem Abstand erfolgen. Tritt während der Kontrollen eine erneute Zunahme des Makulaödems aufgrund des Verschlusses oder ein ödembedingter Verlust der Sehschärfe ein, so sollte erneut behandelt werden (• Abb. 4a; [7]).

Die Therapie sollte abgebrochen werden, wenn

- ein weiterer positiver Behandlungsverlauf nicht mehr zu erwarten ist (z. B.: ausgedehnte RPE-Atrophie, Atrophie der äußeren Netzhaut),

- trotz Rückbildung des Ödems im SDOCT-Volumenscan kein Visusanstieg eingetreten ist,

- die Therapie keinen positiven Einfluss auf Ödem und Visus nimmt [7].

Besteht ein visusminderndes Makulaödem aufgrund eines retinalen venösen Verschlusses, so wird in der Stellungnahme der Fachgesellschaften die bedarfsorientierte intravitreale Anti-VEGFInjektion als relativ risikoarmes Verfahren
Im Falle eines ausbleibenden positiven Einflusses auf das Makulaödem oder den Visus kann ein Therapiewechsel erwogen werden [7]. 

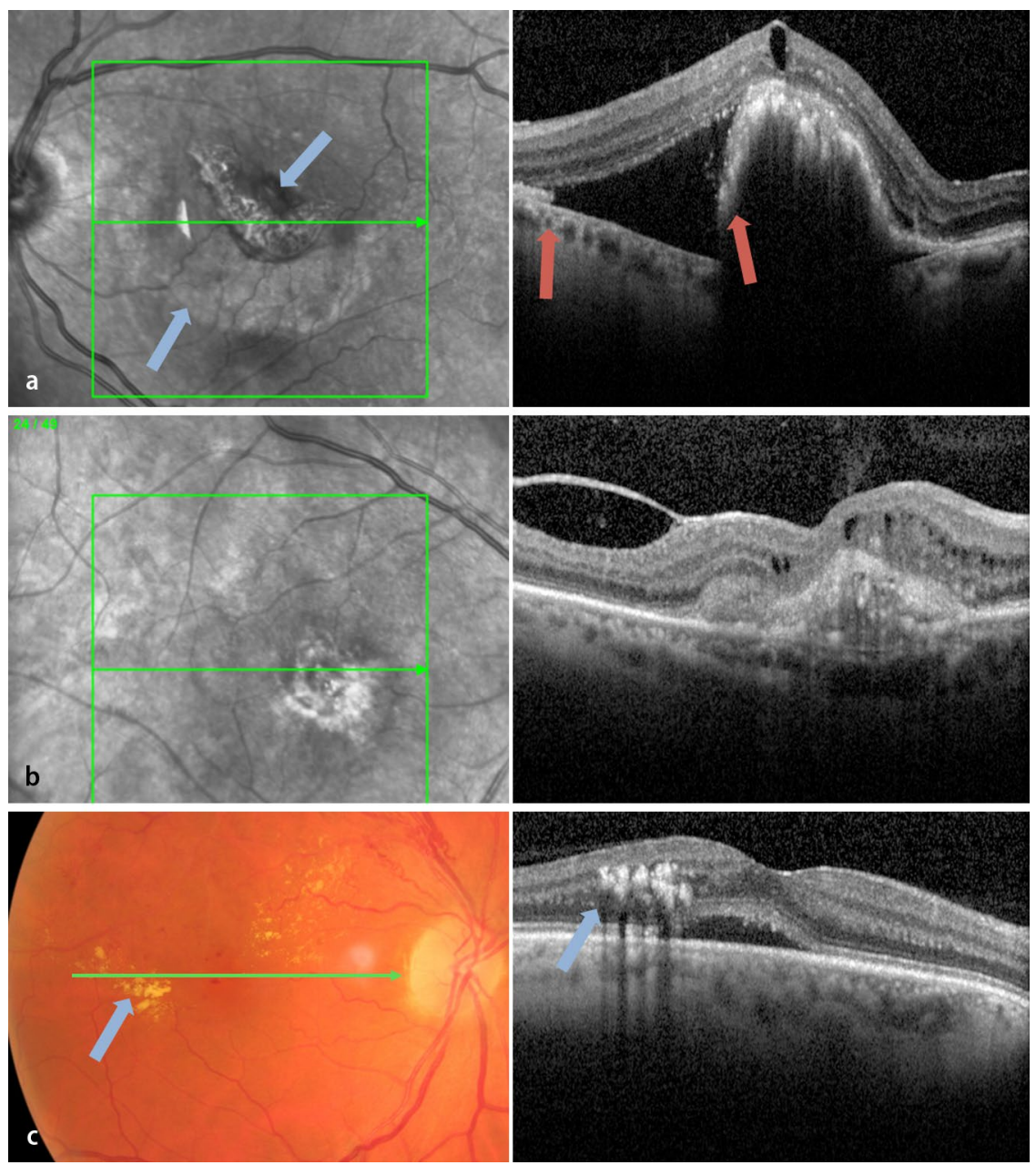

Abb. $3 \Delta$ a Darstellung eines Risses im Pigmentepithel (RIP) im Infrarotbild links und OCT rechts; der obere blaue Pfeil kennzeichnet dass aufgerollte retinale Pigmentepithel (RPE), der untere die dadurch entstehende Atrophie; rechts kennzeichnen die roten Pfeile das Fehlen eines RPE im RIP-Bereich; aufgenommen mit Spectralis-OCT. b Darstellung einer choroidalen Neovaskularisation mit zusätzlicher Glaskörperadhäsion und minimaler Traktion; aufgenommen mit Spectralis-OCT. c Lipidablagerungen (blaue Pfeile) im Fundusfoto links und SD-OCT rechts; mit Spectralis-OCT aufgenommen

\section{Teilnahmekriterien}

Voraussetzungen für den Einschluss als Studienzentrum in das ORCA-Modul waren die Möglichkeit der Durchführung von SD-OCT-Untersuchungen sowie die routinemäßige Einhaltung von Qualitätsstandards.

\section{Empfehlung zu \\ bildgebenden Verfahren}

Die Empfehlungen zu Aufnahmeparametern und Protokollen der bildgebenden Verfahren für das ORCA-Modul wurden anhand der Stellungnahmen der Fachgesellschaften, aktueller Literatur sowie internationaler Standards von den
3 Reading-Centern erarbeitet. Ziel ist es, eine hinreichende Qualität der Aufnahmen zu gewährleisten, die eine hochwertige Auswertung der Bilder bei gleichzeitiger Berücksichtigung der praktischen Durchführung in der klinischen Betreuung von Patienten in der Routine (d. h. außerhalb von randomisierten Studien) ermöglicht.

Nachfolgend sind einige Parameter für die wesentlichen Untersuchungen dargestellt.

\section{Fundusfotografie und Fluoreszeinangiographie}

Wie eingangs beschrieben, umfasst die Basisdiagnostik aller 3 Indikationen im ORCA-Modul zusätzlich zu der SD-OCT-
Untersuchung auch eine Fundusfotografie und Fluoreszeinangiographie. Für Letztere kann sowohl eine Funduskamera als auch ein konfokales Scanning-LaserOphthalmoskop eingesetzt werden. Eine Angiographie ist weiterhin immer dann sinnvoll, wenn ein Befund durch SDOCT alleine nicht erklärt werden kann oder Unsicherheiten bei der SD-OCTBefunderstellung bestehen.

Für die nvAMD sollte der Aufnahmewinkel $30-40^{\circ}$ betragen, bei den anderen Diagnosen sind Weitwinkelaufnahmen $\left(50-60^{\circ}\right)$ empfohlen. Folgende Felder sollten aufgenommen werden (• Abb. 5):

- Feld 1M: temporaler Rand der Papille befindet sich im Bildzentrum.

- Feld 2: Fovea befindet sich im Bildzentrum.

- Feld 3M: Fovea befindet sich ca. 1 Papillendurchmesser vom Bildrand.

Die Fundusfotos sollten eine Fundusreflexaufnahme enthalten sowie die genannten Felder mit jeweils weißem und rotfreiem Licht. Stereoskopische Aufnahmen werden im klinischen Alltag meist nicht durchgeführt und sind nicht erforderlich.

Aufnahmen der Angiographie erfolgen bei Start und Ende der Injektion sowie in der Frühphase (bis $60 \mathrm{~s}$ ), Mittelphase (1-3 min) und Spätphase (5-10 min) der Angiographie. Bei der diabetischen Makulopathie sowie einem Makulaödem bei RVV sind in der Mittelphase zusätzlich periphere Aufnahmen in allen 4 Quadranten erforderlich (• Abb. 5).

Bei diabetischen Veränderungen sollten auch am Partnerauge in der Mittelphase periphere Aufnahmen gemacht werden, bei nvAMD und RVV reicht eine 3-Feld-Aufnahme des Partnerauges aus.

\section{SD-OCT-Untersuchung}

Folgende SD-OCT-Instrumente wurden zur Diagnostik der makulären Veränderungen in der Studie zugelassen:

- Spectralis-OCT von Heidelberg Engineering,

- 3D-OCT-1000/2000 von Topcon,

- Cirrus HD-OCT 400/4000 oder 500/5000, CIRRUS photo 600/800 von Carl Zeiss Meditec sowie - SOCT Copernicus von Optopol. 

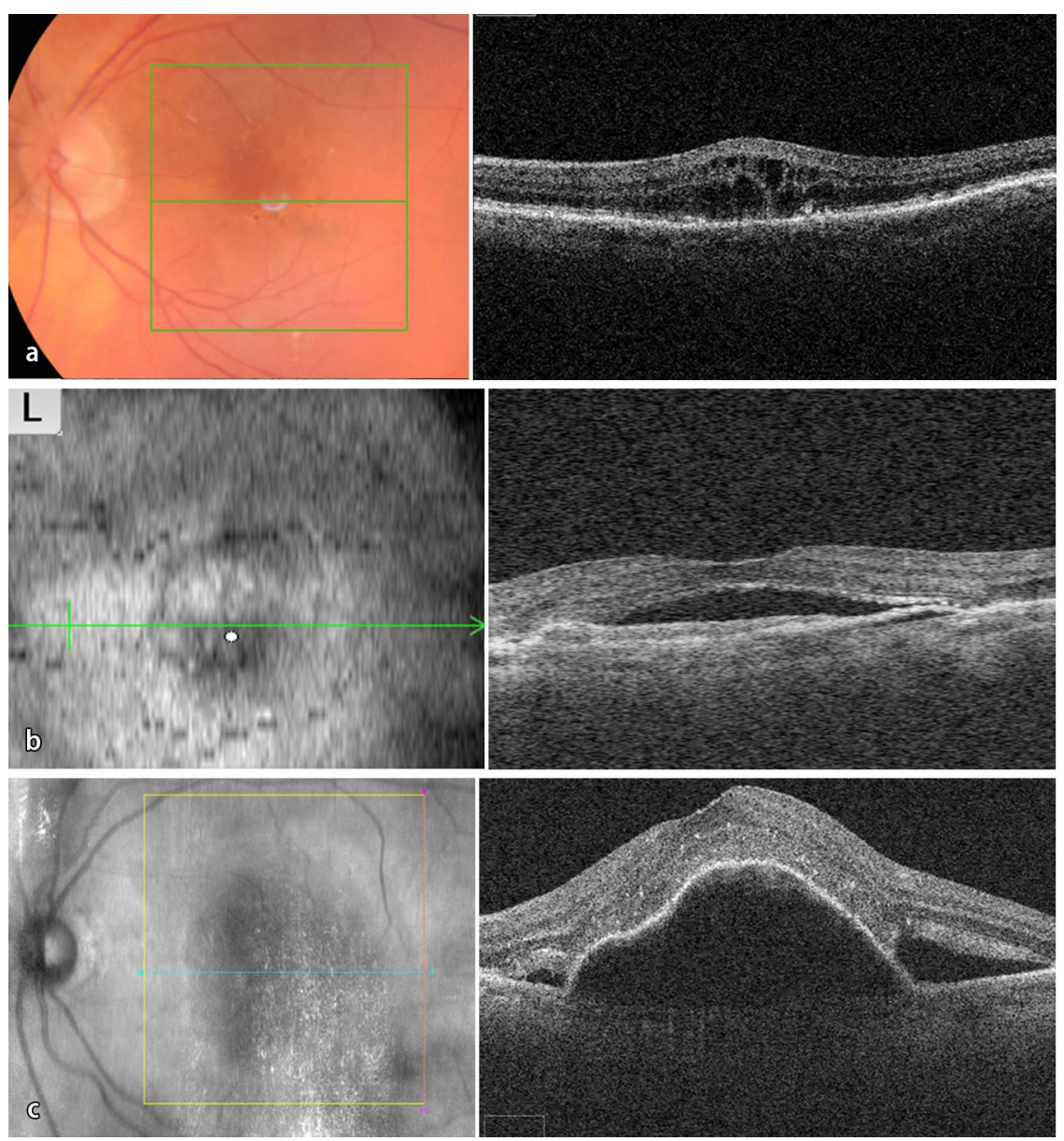

Abb. $4 \Delta$ a Darstellung zystoider, intraretinaler Flüssigkeit; Beispiel aufgenommen mit Topcon 3DOCT. b Beispiel subretinaler Flüssigkeit; aufgenommen mit Copernicus-OCT. c Beispiel einer Pigmentepithelanhebung (Mitte) mit umgebend subretinaler Flüssigkeit; aufgenommen mit Cirrus-OCT

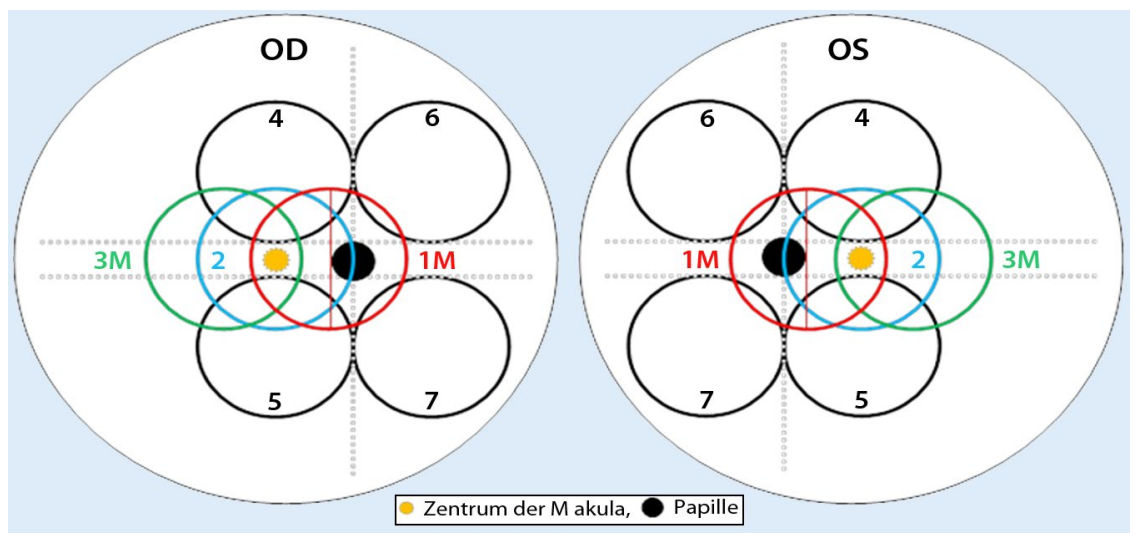

Abb. 5 A Schwarzer Fleck = Papille, gelber Fleck = Makula. Feld 1M: temporaler Rand der Papille im Bildzentrum, Feld 2: Fovea im Bildzentrum, Feld 3M: Fovea ca. 1 Papillendurchmesser zum Bildrand. Periphere Aufnahmen: Feld 4: superior temporal, Feld 5: inferior temporal, Feld 6: superior nasal; Feld 7: inferior nasal. OD rechtes Auge, OS linkes Auge

Da alle Geräte unterschiedliche Module anbieten, sind auch die qualitativen Mindestanforderungen an den Volumenscan jedes Gerätes unterschiedlich definiert (• Tab. 1).
Das Spectralis-OCT sowie das Cirrus HD-OCT (seit dem Update 6.x) besitzen eine Eye-Tracker-Funktion, die es ermöglicht, den OCT-Scanner bei Augenbewegungen nachzuführen [13]. Dies er- möglicht die genaue Zuordnung des OCT-Scans im Fundus sowie Verlaufskontrollen, bei denen die einzelnen Scans an der gleichen Stelle liegen [12].

\section{Grading-Parameter}

Die 3 Reading-Center haben einen Fragebogen entwickelt, der Fragen zur Diagnose sowie zu sämtlichen Parametern enthält, die relevant für die verschiedenen durch die Fachgesellschaften sowie internationale Literatur definierten (Wieder-)Behandlungs- und Abbruchkriterien sowie prognostisch relevante Faktoren sind $[6-9,15]$.

Der Fragebogen wird unabhängig voneinander sowohl durch ärztliche Mitarbeiter (sog. „Reader“) der ReadingCenter als auch durch den an der Studie teilnehmenden Augenarzt bei jeder Visite innerhalb des 24-monatigen Studienzeitraumes ausgefüllt. Der Augenarzt dokumentiert zusätzlich das Visitendatum, Datum und Art einer Behandlung sowie die Sehschärfe. Die Reading-Center beurteilen zusätzlich die Qualität der Bilder.

Bei der Basisvisite wird anhand aller verfügbaren bildgebenden Befunde die Diagnose gestellt, und es erfolgt die Einschätzung, ob eine Anti-VEGF-Therapie sinnvoll ist.

Basierend auf Fundusfarbaufnahmen und Angiographiebildern, werden intraoder subretinale oder sub RPE gelegene Blutungen und deren Bezug zur Fovea (bei nvAMD) sowie eine Glaskörperblutung (bei DR) erfasst. Bei Patienten mit nvAMD wird bei den Folgevisiten zusätzlich dokumentiert, ob ein Pigmentepithelriss eingetreten ist ( $\bullet$ Abb. 3a).

Die SD-OCT-Befunderhebung umfasst das Vorhandensein von intraretinaler Flüssigkeit, intraretinalen zystoiden Räumen (• Abb. 4a), subretinaler Flüssigkeit (• Abb. 4b) sowie die Einschätzung, ob eine foveale Beteiligung vorliegt. Weiterhin wird erhoben, ob eine Pigmentepithelabhebung (PED) vorhanden ist (- Abb. 4c). Bei Folgevisiten wird evaluiert, ob eine Zunahme oder Abnahme der Befunde oder ein stabiler Befund vorliegt.

Die Netzhautdicke wird direkt in der Fovea („foveal central point", FCP) und 


\begin{tabular}{|c|c|c|c|c|c|c|c|}
\hline Gerät & Scan-Modus & Anzahl A-Scans & Anzahl B-Scans & $\begin{array}{l}\text { Abstand B- } \\
\text { Scans }(\mu \mathrm{m})\end{array}$ & $\begin{array}{l}\text { Scan-Maße } \\
(\mathrm{mm})\end{array}$ & Signalstärke & Eye-Tracker \\
\hline Spectralis-OCT & Volume Scan & 512 & $49^{a}$ & Etwa 120 & $6 \times 6$ & & $\mathrm{Ja}$ \\
\hline Topcon-3D-OCT & 3D-Scan & 512 & 128 & Etwa 47 & $6 \times 6$ & $\geq 50$ & Nicht möglich \\
\hline Cirrus-OCT & Macular Cube & 512 & 128 & Etwa 47 & $6 \times 6$ & $\geq 6 / 10$ & $\mathrm{~b}$ \\
\hline Copernicus-OCT & Retina & 512 & 49 & Etwa 120 & $6 \times 6$ & & Nicht möglich \\
\hline
\end{tabular}

im Bereich der zentralen $1000 \mu \mathrm{m}$ (,foveal central subfield“, FCS) getrennt voneinander bestimmt $(\bullet A b b .6 a$ und $b)$. Diese Auswertung erfolgt dabei in der gerätespezifischen Software. Für die Dickenbestimmung am FCP wird - um einen einheitlichen Standard zwischen den verschiedenen SD-OCT-Geräten annähernd zu erreichen - die Netzhautdicke als der Abstand zwischen vitreoretinalem Interface (innere Grenze) und BruchMembran (äußere Grenze) definiert. Für die Bestimmung der FCS wird zunächst überprüft, ob der jeweilige automatisierte Algorithmus der SD-OCT-Software die Netzhautgrenzen nach Ermessen des Auswerters richtig erkannt hat. Außerdem wird die Zentrierung des Gitters bestätigt. Wird bei einem dieser beiden Punkte ein Fehler festgestellt, so ist auch die automatisch gemessene Netzhautdicke fehlerhaft. Wird eine Fehlmessung festgestellt, so werden die Segmentierungslinien bzw. die Position des Grids ggf. manuell korrigiert. Insbesondere die Segmentierung der Netzhautdicke ist für die Bestimmung der FCS-Dicke aufwendig, da hier alle einzelnen B-Scans innerhalb des zentralen Feldes auf das mögliche Auftreten von Artefakten in der automatischen Segmentierung überprüft und diese ggf. korrigiert werden müssen. Zusätzlich ist zu beachten, dass bisher kein Industriestandard für die Netzhautdickenbestimmung mittels SD-OCT-Bildgebung etabliert ist, insbesondere beruht die automatische Segmentierung der Netzhautdicke auf unterschiedlichen Definitionen der äußeren Grenze der Netzhaut. In der ORCA-Studie beruht daher auch die Bestimmung der Netzhautdicke im FCS auf der jeweils gerätespezifischen Definition. Bei ungenauer Zentrierung des ETDRSGrid und/oder ungenauer Segmentierung muss der Untersucher nach eigenem Er- messen entscheiden, ob eine Korrektur "zu aufwendig“, „nicht möglich“ oder sinnvoll erscheint. Im letzteren Fall muss er anschließend die Korrektur durchführen, und ein entsprechender Messwert wird erfasst.

Zuletzt wird im Fragebogen erfasst, wie der Arzt die Prognose einschätzt, d. h., ob bei der vorliegenden morphologischen Situation ein weiterer positiver Behandlungsverlauf zu erwarten ist. Außerdem wird erfasst, wie sicher sich der Arzt - nach seiner eigenen Einschätzung - bei der vorliegenden Beurteilung der SD-OCT-Befunde war.

\section{Auswertung}

Die Befundauswertung durch die Studienärzte wird mit der Auswertung durch die Reading-Center von dritter Seite (unabhängiger Statistiker) verglichen.

Der primäre Endpunkt der Studie ist die Übereinstimmung in der Bewertung von mindestens einem OCTAktivitätskriterium. Stratifiziert wird diese Analyse anschließend nach Diagnose und nach der Art der Visiten (Basisvisite, Folgevisite, Abschlussvisite).

Sekundäre Endpunkte umfassen u. a.:

- Übereinstimmung bezüglich

qualitativer und quantitativer

(Wieder-)Behandlungs- und Abbruchkriterien gemäß den Stellungnahmen der Fachverbände,

- Übereinstimmung in der quantitativen Berechnung der Netzhautdicke für das FCS und den FCP,

- Anzahl der Fälle, in denen eine mögliche Unterbehandlung erfolgte,

- Anzahl der Fälle, bei denen eine Wiederbehandlung bereits ohne SDOCT-Untersuchung indiziert gewesen ist,
- Vergleich der Visusentwicklung von Patienten, bei denen SD-OCTAktivitätskriterien übersehen wurden, zu Patienten, bei denen korrekt befundet wurde.

\section{Qualitätssicherung}

Die Auswertung durch die ReadingCenter erfolgt verblindet in Bezug auf Befunderhebung oder Behandlung durch die Studienärzte. Dies gewährleistet eine unabhängige Bewertung der Aufnahmen.

Die Auswertung in den ReadingCentern erfolgt anhand eines speziell für diese Studie entworfenen Auswertungsprotokolls. Alle an der Auswertung beteiligten Reader sind Ärzte in Weiterbildung oder Fachärzte für Augenheilkunde. Vor Bearbeitung von Studienvisiten, muss sich jeder Reader mit dem Grading-Handbuch vertraut machen und selbstständig 25 Beispielvisiten bearbeiten. Dieses Training erfolgt unter Anleitung eines Senior-Readers (Facharzt für Augenheilkunde).

Nach der Bearbeitung einer Studienvisite von 2 unabhängigen Readern aus jeweils verschiedenen Reading-Centern erfolgt eine finale Auswertung durch einen Senior-Reader ( $\bullet$ Abb. 1). Dieser entscheidet bei diskrepanten Antworten, welches die richtige Alternative ist, und hat auch die Möglichkeit, unzutreffende Angaben zu korrigieren und zu überstimmen (Mixed-arbitration-review-Analyse).

Jede zehnte Visite wird darüber hinaus durch Senior-Reader aus allen 3 ReadingCentern bewertet, um eine zentrumsübergreifende einheitliche Auswertung der bildgebenden Befunde zu gewährleisten und einen zeitlichen Drift zu vermeiden.

Intermittierend werden zudem Aufnahmen durch alle 3 Reading-Center be- 

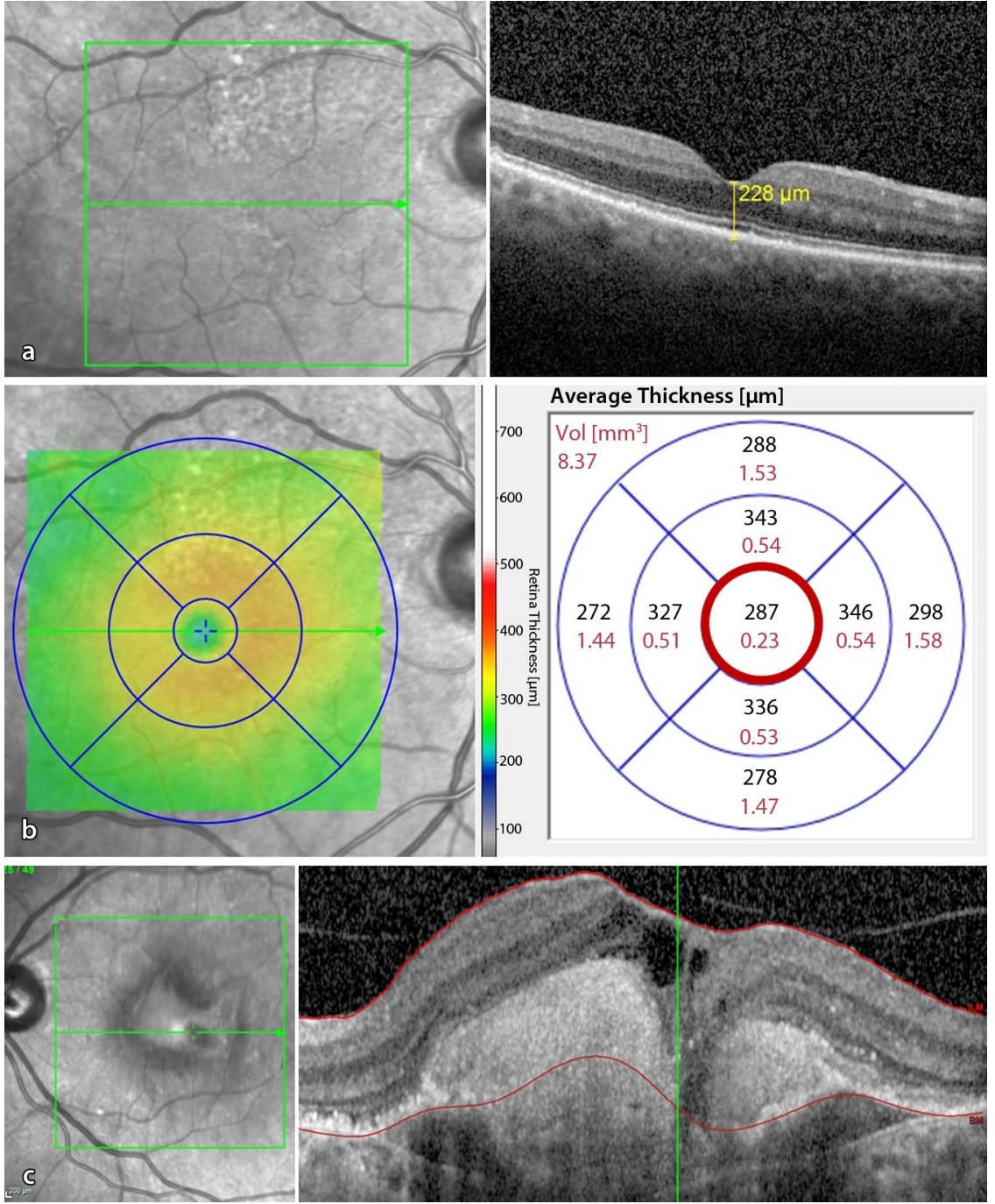

Abb. 6 - Messung der a foveolären Netzhautdicke (FCP) sowie b der mittleren Netzhautdicke im zentralen Unterfeld mit $1000 \mu \mathrm{m}$ Durchmesser (FCS), rot markiert. c Voraussetzung ist eine korrekte Lage der Segmentierungslinien. In diesem Beispiel folgt die innere Segmentierungslinie korrekt der inneren Grenzmembran. Die äußere Segmentierungslinie folgt zum Teil dem retinalen Pigmentepithel, zum Teil läuft sie durch das subretinale hyperreflektive Material. Bedingt durch diese fehlerhafte Segmentierung spiegelt der generierte Dickenwert weder die Netzhautdicke noch die Dicke von Netzhaut + choroidaler Neovaskularisation wider

gutachtet, Unterschiede analysiert und diskutiert.

\section{Diskussion}

Die Bedeutung derSD-OCT-Untersuchung für die Behandlung von Patienten mit nvAMD oder Makulaödem bei DR oder RVV hat in den letzten Jahren erheblich zugenommen. Das ORCA-Modul der OCEAN-Studie ist eine Beobachtungsstudie, die den Versorgungsalltag untersucht mit besonderem Augenmerk auf die Qualität der SD-OCT-Auswertung, die bei der bedarfsorientierten Behandlungsstrategie eine Schlüsselrolle einnimmt.

Die Kenntnis der relevanten SD-OCTParameter sowie eine hohe Sicherheit bei der Interpretation der bildgebenden Befunde sind von zentraler Bedeutung, um eine korrekte Diagnose und somit Therapieindikation stellen zu können, die Prognose einschätzen zu können und möglichst eine Unter- oder Überbehandlung der Patienten zu vermeiden.

Um eine qualitative Auswertung der morphologischen Kriterien zu ermöglichen, wurden konkrete Mindestanforderungen für die Basisdiagnostik definiert. Verschiedene SD-OCT-Geräte wurden in einer Untersuchung auf Reproduzierbarkeit und Präzision untersucht $[1,20]$. Es konnte eine hohe Reproduzierbarkeit der Aufnahmen bei einer axialen Auflösung für die in ORCA eingeschlossenen Geräte zwischen 4 und $6 \mu \mathrm{m}$ erhoben werden $[1,20]$. Trotzdem ist eine Angiographie erforderlich, um den Perfusionsstatus zu erkennen und Ischämien ausschließen, um Differenzialdiagnosen abgrenzen sowie Hyperpermeabilitäten (Leckage) von Anfärbung (Staining) differenzieren zu können. In der Erfahrung der Autoren zeigt sich, dass CNVs mit relativ wenig Flüssigkeit im OCT einhergehen und sich durch eine hyperreflektive, unscharf begrenzte subretinale Substanzvermehrung bemerkbar machen. Auch hier ist eine Angiographie hilfreich, um die Aktivität darzustellen. Die Farbfotografie liefert weitere Informationen über die Lokalisation und Ausdehnung der Läsionen sowie begleitende Veränderungen und ermöglicht auch die Dokumentation von Blutungen.

Um Befunde möglichst präzise und lückenlos erfassen zu können, sollte ein volumetrischer Scan von mindestens $20 \times 20^{\circ}$ oder $6 \times 6 \mathrm{~mm}$ mit Abdeckung des inneren ETDRS-Rasters mit einem Abstand der Schnitte $<188 \mu \mathrm{m}$ erfolgen. Die Papille sollte in der Übersicht möglichst mit erfasst werden und kann so zur Orientierung dienen [6-8].

Nutzt man die Kartierungsfunktion der Geräte, so ist eine relevante Voraussetzung, dass zunächst die Lage der Fovea überprüft wird und das Raster der Herstellersoftware daran ausgerichtet wird (- Abb. 6a bis c). Nachfolgend muss überprüft werden, ob die Segmentierungslinien richtig liegen. Die obere Linie liegt immer auf der inneren limitierenden Membran, während sich die Lage der unteren Linie je nach Herstellersoftware unterscheiden kann und zwischen Photorezeptorniveau und Bruch-Membran variiert. Zwischen diesen beiden Linien erfolgt die eigentliche Messung der Netzhautdicke, die in der Kartenansicht (Map) meist in Form eines ETDRS-Grids ausgegeben wird.

Trotz aller Fortschritte bleiben bei der Verlässlichkeit der Interpretation der OCT-Befunde noch Unsicherheiten. Diese 
können sowohl auf ungeeigneten Aufnahmeparametern, unpassender Nutzung der softwarespezifischen Möglichkeiten zur Analyse (z. B. Karten) oder Fehlinterpretation von Aktivitätskriterien beruhen. Eine korrekte Interpretation von SD-OCT-Scans ist ebenso anspruchsvoll wie bedeutsam für eine optimale Behandlung der Patienten. Unterschiede in der OCT-Befunderhebung zwischen behandelnden Ärzten und dem ReadingCenter in der CATT-Studie verdeutlichen die Notwendigkeit einer Analyse der realen Situation in der täglichen Routine, um Schwierigkeiten bei der Interpretation spezifischer Parameter aufdecken und ggf. durch gezielte Formulierung von Standards oder Fortbildungen die Qualität der Patientenversorgung in Deutschland verbessern zu können.

Das ORCA-Modul der OCEANStudie wird die OCT-Begutachtung durch die Studienärzte im Vergleich zu Reading-Centern analysieren. Ergebnisse liegen aktuell noch nicht vor. Zukünftig soll diese zur Sicherung eines möglichst hohen Qualitätsstandards beitragen.

\section{Schlussfolgerungen}

Parallel zur Weiterentwicklung der bildgebenden Diagnostik sind Mindeststandards zu definieren, mit denen eine unabhängige und verblindete Qualitätssicherung möglich ist. Die ORCA-Studie wird erstmals Daten liefern, die mögliche Schwachstellen aufzeigen und ein gezieltes Training ermöglichen mit dem Ziel, die Anti-VEGF-Therapie im Versorgungsalltag zu optimieren.

\section{Fazit für die Praxis}

\footnotetext{
- Die OCEAN- und ORCA-Studien erlauben eine Einschätzung der Behandlungs- und Befundungsqualität von Patienten unter RanibizumabTherapie im Behandlungsalltag in Deutschland.

- Eine adäquate Durchführung und zuverlässige Bewertung der bildgebenden Verfahren, insbesondere der SD-OCT-Aufnahmen, bilden die Grundlage für eine korrekte Diagnosestellung sowie eine
}

optimale, SD-OCT-gestützte AntiVEGF-Therapie.

- Die Analyse der ORCA-Studie kann dabei helfen, Trainingsbedarf gezielt zu ermitteln und gemeinsam die Behandlung der Patienten zu optimieren.

\section{Korrespondenzadresse}

\section{S. Liakopoulos}

Cologne Image Reading Center, Zentrum für Augenheilkunde

Uniklinik Köln, Kerpener Str. 62, 50924 Köln sandra.liakopoulos@uk-koeln.de

\section{Einhaltung ethischer Richtlinien}

Interessenkonflikt. Diese Untersuchung wurde von der Firma Novartis Pharma GmbH unterstützt. B. Heimes: Unterstützung von Forschungsprojekten und klinischen Studien: Novartis, Allergan, Quark, Acucela; Beratung, Honorare, Reisekosten: Novartis. T. Schick: Beratung, Honorare, Reisekosten: Novartis. C.K. Brinkmann: Unterstützung von Forschungsprojekten und klinischen Studien durch Heidelberg Engineering, Newtricious, Novartis, Zeiss Meditec; Beratung, Honorare, Reisekosten: Heidelberg Engineering, Newtricious, Novartis. A. Wiedon: Mitarbeiterin der Firma Novartis Pharma $\mathrm{GmbH}$. B. Haegele: Mitarbeiterin der Firma Novartis Pharma $\mathrm{GmbH}$. B. Kirchhof: Unterstützung von Forschungsprojekten und klinischen Studien: Novartis; Beratung, Honorare, Reisekosten: Novartis, Pfizer, Bayer. F.G. Holz: Unterstützung von Forschungsprojekten und klinischen Studien: Acucela, Alcon, Allergan, Bayer, Genentech, Heidelberg Engineering, Novartis, Ophthotech, Roche; Beratung, Honorare, Reisekosten: Allergan, Bayer, Genentech, Novartis, Ophthotech; Advisory Boards: Acucela, Allergan, Avalanche, Bayer, Genentech, Heidelberg Engineering, Johnson \& Johnson, Novartis, Ophthotech, Roche. D. Pauleikhoff: Unterstützung von Forschungsprojekten und klinischen Studien: Novartis, Ophthotech, Acucela, Genentech, Roche, Bayer; Beratung, Honorare, Reisekosten: Novartis, Bayer, Allergan; Advisory Boards: Novartis, Bayer, Allergan. F. Ziemssen: Unterstützung von Forschungsprojekten und klinischen Studien: Allergan, Novartis; Beratung, Honorare, Reisekosten: Alimera, Allergan, Bayer HealthCare, Biogen, Heidelberg Engineering, Novartis; Advisory Boards: Alimera, Allergan, Bayer HealthCare, Novartis. S. Liakopoulos: Beratung, Honorare, Reisekosten: Novartis, Bayer, Heidelberg Engineering; Advisory Boards: Novartis. G. Spital: Unterstützung von Forschungsprojekten und klinischen Studien: Novartis, Ophthotech, Pfizer, Acucela, Genentech, Roche, Bayer; Beratung, Honorare, Reisekosten: Allergan, Bayer, Novartis; Advisory Boards: Allergan, Alimera Science, Bayer, Novartis. S. Schmitz-Valckenberg: Unterstützung von Forschungsprojekten und klinischen Studien durch Allergan, Bayer, Formycon, Genentech, Heidelberg Engineering, Novartis, Optos, Roche, Topcon, Zeiss Meditec; Beratung, Honorare, Reisekosten: Alcon, Alimera, Bayer, Heidelberg Engineering, Novartis, Optos.
Alle im vorliegenden Manuskript beschriebenen Untersuchungen am Menschen wurden mit Zustimmung der zuständigen Ethik-Kommission, im Einklang mit nationalem Recht sowie gemäß der Deklaration von Helsinki von 1975 (in der aktuellen, überarbeiteten Fassung) durchgeführt. Von allen beteiligten Patienten liegt eine Einverständniserklärung vor.

Open Access Dieser Artikel unterliegt den Bedingungen der Creative Commons Attribution License. Dadurch sind die Nutzung, Verteilung und Reproduktion erlaubt, sofern der/die Originalautor/ en und die Quelle angegeben sind.

\section{Literatur}

1. Bentaleb-Machkour Z, Jouffroy $E$, Rabilloud M et al (2012) Comparison of central macular thickness measured by three OCT models and study of interoperator variability. ScientificWorldJournal 2012:842795

2. Bloch SB, Larsen M, Munch IC (2012) Incidence of legal blindness from age-related macular degeneration in denmark: year 2000 to 2010 . Am J Ophthalmol 153:209-213

3. Bunce C, Xing W, Wormald R (2010) Causes of blind and partial sight certifications in England and Wales: April 2007-March 2008. Eye 24:1692-1699

4. Campbell JP, Bressler SB, Bressler NM (2012) Impact of availability of anti-vascular endothelial growth factor therapy on visual impairment and blindness due to neovascular age-related macular degeneration. Arch Ophthalmol 130:794-795

5. Catt Research Group (2011) Ranibizumab and bevacizumab for neovascular age-related macular degeneration. N Engl J Med 364(20):1897-1908

6. Deutsche Ophthalmologische Gesellschaft, Retinologische Gesellschaft, Berufsverband Der Augenärzte Deutschlands (2015) Die Anti-VEGFTherapie bei der neovaskulären altersabhängigen Makuladegeneration: Therapeutische Strategien. Ophthalmologe 112:237-245

7. Deutsche Ophthalmologische Gesellschaft, Retinologische Gesellschaft, Berufsverband Der Augenärzte Deutschlands (2012) Stellungnahme zur Therapie des Makulaödems beim retinalen Venenverschluss. Ophthalmologe 109:818-831

8. Deutsche Ophthalmologische Gesellschaft, Retinologische Gesellschaft, Berufsverband Der Augenärzte Deutschlands (2013) Stellungnahmen zur Therapie der diabetischen Makulopathie und Anwendung von Ocriplasmin (JETREA ${ }^{\oplus}$ ) in der Augenheilkunde. Ophthalmologe 110:568-588

9. Ferris FL 3rd, Wilkinson CP, Bird A et al (2013) Clinical classification of age-related macular degeneration. Ophthalmology 120(4):844-851

10. Finger RP, Bertram B, Wolfram C et al (2012) Blindness and visual impairment in Germany: a slight fall in prevalence. Dtsch Arztebl Int 109:484489

11. Finger RP, Wiedemann $P$, Blumhagen $F$ et al (2013) Treatment patterns, visual acuity and quality-of-life outcomes of the WAVE study - a noninterventional study of ranibizumab treatment for neovascular age-related macular degeneration in Germany. Acta Ophthalmol 91(6):540-546

12. Heidelberg Engineering Gmbh (2013) AutoRescan. http://www.heidelbergengineering. com/germany/produkte/spectralis/wichtigefunktionen/autorescan/. Zugegriffen: 26. Juli 2013 
13. Heidelberg Engineering Gmbh (2013) TruTrack Aktives Eye Tracking. http://www.heidelbergengineering.com/germany/produkte/spectralis/ technologie/trutrack-aktives-eye-tracking/. Zugegriffen: 26. Juli 2013

14. Holz FG, Tadayoni R, Beatty S et al (2015) Multicountry real-life experience of anti-vascular endothelial growth factor therapy for wet agerelated macular degeneration. $\mathrm{Br} J$ Ophthalmol 99(2):220-226

15. Keane PA, Patel PJ, Liakopoulos S et al (2012) Evaluation of age-related macular degeneration with optical coherence tomography. Surv Ophthalmol 57:389-414

16. Martin DF, Maguire MG, Fine SL et al (2012) Ranibizumab and bevacizumab for treatment of neovascular age-related macular degeneration: two-year Results. Ophthalmology 119:1388-1398

17. Pruente C, Study-Group R (2014) Efficacy and safety of ranibizumab in two treat-and-extend versus pro-re-nata regimes in patients with visual impairment due to diabetic macular edema: 24-month results of RETAIN study. http://www. arvo.org/webs/am2014/abstract/sessions/236.pdf. Zugegriffen: 5. Dez. 2014

18. Rush RB, Simunovic MP, Aragon AV 2nd et al (2014) Treat-and-extend intravitreal bevacizumab for branch retinal vein occlusion. Ophthalmic Surg Lasers Imaging Retina 45:212-216

19. Skaat A, Chetrit A, Belkin M et al (2012) Time trends in the incidence and causes of blindness in Israel. Am J Ophthalmol 153:214-221

20. Wolf-Schnurrbusch UE, Ceklic L, Brinkmann CK et al (2009) Macular thickness measurements in healthy eyes using six different optical coherence tomography instruments. Invest Ophthalmol Vis Sci 50:3432-3437

21. Wolf A, Kampik A (2014) Efficacy of treatment with ranibizumab in patients with wet age-related macular degeneration in routine clinical care: data from the COMPASS health services research. Graefes Arch Clin Exp Ophthalmol 252(4):647-655

22. World Health Organization (2002) Prevention of Blindness and Visual Impairment: Causes of blindness and visual impairment. http://www. who.int/blindness/causes/en/. Zugegriffen: 03. Mai 2013

23. Zweifel SA, Engelbert M, Laud K et al (2009) Outer retinal tubulation: a novel optical coherence tomography finding. Arch Ophthalmol 127:1596-1602

\section{Immer aktuell - Dank Online-First}

\section{Warum warten, bis die Artikel im Heft abgedruckt sind, wenn Sie sie auch schon vorher lesen können?}

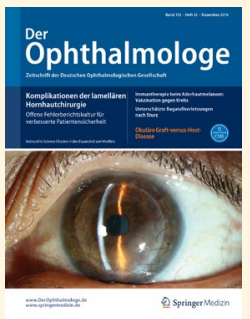

Lesen Sie unter

www.springermedizin.de/der-ophthalmologe

Reiter "Online First Artikel”

die aktuellsten Artikel, die gerade online veröffentlicht wurden.

Folgende Übersichten, Originalien, Kasuistiken und „Bild und Fall“-Beiträge wurden im vergangenen Monat online publiziert:

— Konfokale Mikroskopie als früher Rezidivmarker nach Keratoplastik

infolge einer Fusarium-solani-Keratitis.

Daas L, Bischoff-Jung M, Viestenz A, Seitz B, Viestenz A.

DOI 10.1007/s00347-016-0270-4

- Zentralarterienverschluss nach stumpfem Bulbustrauma.

Filev F, Atiskova Y, Klemm M.

DOI 10.1007/s00347-016-0274-0

— Bilaterale kristalline Einlagerungen im Hornhautstroma.

Auerbach FN, Khoramnia R, Auffarth GU, Hernandez J, Tandogan T, Sel S.

DOI 10.1007/s00347-016-0269-x

- Glaskörpermetastase eines malignen Melanoms der Haut unter Ipilimumab. Kellermann N, Maier M, Feucht N.

DOI 10.1007/s00347-016-0271-3

- Bilaterale Netzhautabhebungen.

Melzer CA, Schmitz-Valckenberg S, Holz FG.

DOI 10.1007/s00347-016-0277-x

- Hornhautspende - Ein Dilemma zwischen wachsender Nachfrage und sinkender Spenderrate. Schaub F, Simons HG, Enders P, Bachmann B, Roters S, Cursiefen C, Heindl LM.

DOI 10.1007/s00347-016-0281-1

— Unilaterale Photophobie als Erstsymptom einer schweren Allgemeinerkrankung.

Marjanovic I, Löw U, Seitz B.

DOI 10.1007/s00347-016-0255-3

- Compliance von AMD-Patienten unter Anti-VEGF-Therapie-Analyse

und Verbesserungsvorschläge.

Heimes B, Gunnemann F, Ziegler M, Gutfleisch M, Spital G, Pauleikhoff D, Lommatzsch A.

DOI 10.1007/s00347-016-0275-z

- Iritis - Was hat das Glaukom damit zu tun?

Hopf S, Prokosch V, Bornscheuer C, Pfeiffer N.

DOI 10.1007/s00347-016-0279-8

— Retinopathia solaris - Eine Fallserie nach der Sonnenfinsternis am 20.03.2015.

Bachmeier I, Helbig H, Greslechner R.

DOI 10.1007/s00347-016-0286-9

— „Bindehaut-Dehiszenz" nach vitreo-retinaler Chirurgie.

Duisdieker V, Herwig MC, Gagalick A, Charbel Issa P, Holz FG, Löffler KU.

DOI 10.1007/s00347-016-0290-0

Übrigens: Wussten Sie schon, dass die Artikel mit Angabe der DOI (digital object identifier) und dem Zusatz „epub ahead of print" bereits zitiert werden können? 